\title{
Inconsistent individual personality description eliminates the other-race effect
}

\author{
Lanya Zhang • Guomei Zhou $\cdot$ Xiaoping Pu • \\ William G. Hayward
}

Published online: 7 July 2011

(C) Psychonomic Society, Inc. 2011

\begin{abstract}
The present study investigated the effect of individual semantic information displayed simultaneously with faces on the other-race effect. Arbitrary descriptions of the individual personalities of a set of faces were initially evaluated for consistency. Later, 83 naïve participants were allocated to three groups in which they saw faces and consistent, inconsistent, or neutral personality information about each face. Later, they completed a recognition task for the faces. The other-race effect was observed only in the control group and the consistent-information group, but not in the inconsistent-information group. This showed that inconsistent individual semantic information for each face can help to individuate these faces and eliminated the other-race effect.
\end{abstract}

Keywords Own-race effect · Individuation .

Consistent/inconsistent description - Semantic information

Humans live in a complex and flexible social context, so the ability of distinguishing "who is who" is important for people to get along well with other social members. Faces are a vital stimulus for this task and provide various types of information (such as gender, age, race, expressions, etc.) to observers. Overall, people are experts at recognizing faces; however, not all the faces are recognized equally. One of the deficits of the recognition process is that we recognize faces of unfamiliar races less accurately than those of our own race, and this

L. Zhang $\cdot$ G. Zhou $(\bowtie) \cdot X . P u$

Department of Psychology, Sun Yat-sen University,

135 Xingang West Road,

Guangzhou 510275, China

e-mail: zhougm@mail.sysu.edu.cn

W. G. Hayward

Department of Psychology, The University of Hong Kong,

Pokfulam Road,

Hong Kong, China phenomenon is called the other-race effect (ORE; also referred to as the cross-race effect or own-race advantage; Malpass \& Kravitz, 1969; Meissner \& Brigham, 2001). Many theories have been proposed to explain this phenomenon, and most of them can be grouped into two different kinds of theoretical models: perceptual-expertise models and social-categorization models (see Bernstein, Young, \& Hugenberg, 2007).

Perceptual-expertise models regard an observer's differential expertise for same-race and cross-race faces as the critical cause of the ORE. A considerable body of research supports this standpoint. For instance, researchers found that cross-race identification accuracy was positively correlated with selfreported interracial contact (Hancock \& Rhodes, 2008; Wright, Boyd, \& Tredoux, 2003); adoption studies have shown that if children have been adopted by people from another race and live in another country, they will show a reversal of the ORE by adulthood (Sangrigoli, Pallier, Argenti, Ventureyra, \& de Schonen, 2005). Michel, Caldara, and Rossion (2006) and Tanaka, Kiefer, and Bukach (2004) even found that such experience could affect the processing style of faces: Caucasians showed a larger holistic-processing advantage for own-race faces than for other-race faces, whereas Asians showed an equally large holistic-processing advantage for both races. These results were explained as being due to experience; Asians living in Caucasiandominated countries generally have experience with faces of both races, whereas Caucasians tend to have differentially more experience with Caucasian faces. Although there is considerable empirical support for perceptual-expertise models, some researchers have argued that there are also socialcognitive mechanisms underlying the ORE.

Sporer (2001) suggested that the inadequacy in recognizing other-race faces was because people categorize them as out-group members, using the cue of race. This categorization cue may lead observers to allocate less effort to encoding other-race faces or may lead to shallow 
encoding of such faces. More recently, Hugenberg and his colleagues (Hugenberg, Miller, \& Claypool, 2007; Hugenberg \& Sacco, 2008; Hugenberg, Young, Bernstein, $\&$ Sacco, 2010) further proposed the categorizationindividuation model (CIM). The core assumption of the CIM is that perceivers individuate in-group members; however, they simply think about out-group members in terms of categories. Categorizing out-group members reduces recognition performance, and individuating in-group members elicits an in-group memory advantage. Because thinking about out-group members in terms of categories hampers memory, individuating out-group members is a counterweight solution to overcoming the memory deficits of out-group face memory (Hugenberg \& Sacco, 2008).

However, so far there are only a few studies supporting the individuating advantage, and most of them do not directly manipulate individuation processing. For instance, Hugenberg, Miller, and Claypool (2007) manipulated encoding instructions to elicit individuation of out-group members and found that the ORE was eliminated. Specifically, the individuation group was informed of the existence of the ORE and was asked to pay close attention to and try especially hard to learn the other-race faces to avoid the ORE. As predicted, the ORE showed up in the control group but disappeared in the individuation group. The alternate explanation of a broad motivation to process each face, instead of the individuation instruction, was ruled out by their second experiment, in which a third group that was asked to pay very close attention to each face still showed the ORE.

Some other studies have also indirectly supported the CIM. For example, Johnson and Fredrickson (2005) found that positive emotions in perceivers eliminated the ORE. The authors suggested that positive emotion may make perceivers more open-minded and flexible, which can help them individuate other-race faces, rather than just categorizing them. Ackerman, Shapiro, Neuberg, Kenrick, Becker, Griskevicius, and Schaller (2006) found that white perceivers remembered angry black faces better than angry white faces. They argued that the angry expression of other-race faces is a threatening cue, which will trigger danger-relevant stereotypes about other-race members. Then self-protection may be activated by out-group members, who are potentially stereotyped as dangerous, and more resources are devoted to individuating them. More recently, Shriver and Hugenberg (2010) showed white participants black and white faces paired with descriptions of their behaviors that conveyed power or submissiveness. They found that high-power black faces, but not low-power black faces, were remembered just as well as white faces. These results suggest that powerful other-race faces tend to be individuated because they are goal relevant (Rodin, 1987; Wright et al., 2003).

A possible perceptual basis for CIM was found by Lebrecht, Pierce, Tarr, and Tanaka (2009), who trained
Caucasians to individuate or categorize African American faces or Chinese faces. In the individuation training condition, participants individuated other-race faces with particular letters such as "a," "s," and "d." In the categorization condition, participants categorized other-race faces by pressing "o." After five sessions of training, the typical old/ new recognition test showed improved performance for the individuation group, but not for the categorization group.

In the present study, we investigated whether individual semantic information for other-race faces can improve participants' memory performance for them and then reduce or eliminate the ORE. We used individual personality descriptions that were either consistent or inconsistent with the corresponding faces as a cue for individuation. To make sure that the individuation manipulation would be reliable, we did not allocate personality information to faces randomly; instead, we developed consistent and inconsistent personality information for each face. Previous studies have shown mixed results for the effect of consistent and inconsistent information on memory. Some researchers have found that consistent semantic information facilitated face memory (Hills, Lewis, \& Honey, 2008; Klatzky, Martin, \& Kane, 1982) and object recognition (Rahman \& Sommer, 2008). However, some other researchers have suggested that highly inconsistent semantic information makes schematic information more memorable (O'Sullivan \& Durso, 1984). For example, participants can remember a "jock" who is physically weak better than one who is physically strong. Other studies (Crocker, Hanna, \& Weber, 1983; Hastie \& Kumar, 1979; Srull, 1981; Srull, Lichtenstein, \& Rothbart, 1985) have shown that people are more likely to recall information when their initial impressions are incongruent with the following information than when the following information is congruent or irrelevant. More recently, Shriver and Hugenberg (2010) found that whether the descriptions of behaviors were stereotypic (e.g., "he punched a person") or counterstereotypic ("he is the CEO of a fortune 500 company") for black faces, other-race (black) faces were remembered just as well as same-race (Caucasian) faces. Therefore, we supposed that both consistent and inconsistent personality information would help to individuate the target faces, as compared with neutral semantic information. However, whether inconsistent information or consistent information would have a stronger effect remained unclear.

In the present study, distinctive personality information for each face, either an own-race or another-race face, was developed in a pilot experiment. In phase 1, participants were asked to select the main consistent and inconsistent personality words for each face. In phase 2, participants were asked to rate the extent to which the personality description below the face described the person, to ensure that the consistent and inconsistent information for each face was reliable. In the formal experiment, participants were required to learn the face together with semantic information. The control group learned the faces 
with neutral semantic information, while the two experimental groups learned the faces with consistent or inconsistent semantic information. We predicted that, as compared with neutral semantic information, the individuated consistent or inconsistent personality information would help to improve other-race face memory and, thus, reduce or eliminate the ORE.

\section{Pilot experiment}

Phase 1: Development of semantic personality stimulus

The first phase was conducted to select consistent and inconsistent personality descriptions for each face.

\section{Method}

Participants Five male and 10 female Chinese students from Sun Yat-sen University took part in the experiment in return for a small payment.

Materials Twenty Caucasian and 20 Chinese male faces from Hayward, Rhodes, and Schwaninger (2008) study were adopted. These faces were printed in $6.00 \times 4.57 \mathrm{~cm}$ areas on the left of A4 papers. On the right area of each paper, adjacent to the face, was the personality word list (see the Appendix), presented in Chinese. The word list for each face was composed of 25 pairs of opposite words from the Big Five Personality Scale (Yang, McCrae, Costa, Dai, Yao, Cai, \& Gao, 1999) and 16 single words that are common descriptors of Chinese personality (Wang \& Cui, 2005). The repeated words appeared only once. There were two faces on each page, totaling 20 pages in all, arranged in a random order for each participant.

Procedure Participants were asked to select 8 words from the list that best described each face. Only 1 word from each pair of the paired bipolar words from the Big-Five Personality Scale was allowed to be selected. In addition, any words from the other 16 single words were allowed to be selected.

\section{Results and discussion}

The personality word with highest frequency for each face was selected for the next phase to develop consistent personality information. Those faces for which the nomination frequencies of all words were lower than six were rerated by another 6 participants. The raters had to rate the top frequency words (using a 1 to 5 scale, where 1 was not appropriate to describe this face at all and 5 was very appropriate to describe this face). The words that got the highest score in rerating were selected as consistent personality information. In addition, one of the words that was never nominated for a particular face was used for the next phase to develop inconsistent personality information.

Phase 2: Development of the stimulus set

We had selected the main consistent and inconsistent personality words for each face in Phase 1 . The goal of Phase 2 was to ensure that the information for each face was reliable.

\section{Method}

Participants and materials Another 16 Chinese students (8 female and 8 male) from Sun Yat-sen University participated in the experiment for payment. All the faces were the same as those in Phase 1.

Design and procedure Each personality word selected in Phase 1 was composed into a sentence, again presented in Chinese, to describe the corresponding face. The pattern of the sentence was "He is a/an __ man." In the blank was the personality word that was consistent or inconsistent with each face.

Two blocks, one for Asian faces and the other for Caucasian faces, were used. The order of the two blocks was counterbalanced across participants. In each block, 40 trials - with 2 trials (one with consistent information, the other with inconsistent information) for each face-were presented randomly. Participants were instructed to rate the extent to which the sentence below the face described the person, using a 5-point Likert scale (1 was not appropriate to describe this face at all; 5 was most appropriate to describe this face), and to press the corresponding number keys. The stimulus was presented on the screen until the participants responded.

\section{Results and discussion}

The consistent description with a mean rating score smaller than 3.00 and the inconsistent description with a mean rating score larger than 3.00 were substituted by other descriptors. Ten more participants rated those faces again with the revised semantic information by following the same procedure, until the rating scores of all the faces with consistent information were higher than 3.00 and of the faces with inconsistent information were lower than 3.00.

The one-sample $t$-test indicated that the consistency ratings for both own- $(M=3.59, S D=0.26)$ and other-race $(M=$ 3.67, $S D=0.26$ ) faces were significantly larger than 3.00, with $t \mathrm{~s}(15)>9.0, p \mathrm{~s}<.001$, and the inconsistency ratings for both own- $(M=2.7, S D=0.31)$ and other-race $(M=2.84$, $S D=0.25)$ faces were significantly lower than 3.00 , with $t$ s $(15)<-2.5, p s<.05$. The results indicated that the consistent 
information and the inconsistent information were reliable. Consequently, they were used in the formal experiment.

\section{Experiment}

Method

Participants Ninety-one Chinese undergraduates (45 female, 46 male) from Sun Yat-sen University in the age group of 1724 years participated in the experiment for payment.

Materials The photographs of the 20 Asian and 20 Caucasian young adult male faces used in the pilot experiments were used in this experiment. These pictures were $5.5 \mathrm{~cm}$ wide $\times 7.4 \mathrm{~cm}$ high and were presented at the center of a black screen. The distance between the participants and the screen was approximately $50 \mathrm{~cm}$.

The semantic information developed in the pilot study was used to describe the faces (see Fig. 1). The pattern of the information was "He is a/an man." The blank was filled by a personality word consistent or inconsistent with this face. The semantic information in the control condition contained neutral information composed of four from 10 familiar household items (in Chinese; ceiling, window, floor, wardrobe, table, chair, sofa, tea table, cupboard, and wall) or four of 10 familiar stationary items (blackboard, ruler, eraser, ink, pen, pencil, book, scissor, desk, and bookshelf). All the words consisted of two or three characters. They were randomly allocated to each face.

All the semantic information was presented in Chinese. The font used for the words was Chinese regular script (Kai Shu) of 14-point size and white color against a black background. The length of every sentence was from 8 to 10 characters. The semantic information was placed $1.5 \mathrm{~cm}$ below the picture of the face.

Design A 2 (race of face: own-race and other-race) $\times 3$ (semantic information: control, consistent, and inconsistent) mixed design was used. Eighty-three participants (26 control, 28 consistent, and 29 inconsistent) were allocated randomly to the three semantic-information conditions and were shown both own-race and other-race faces.

Procedure The participants were informed that this was a face memory study. They received three blocks of trials. The races of the faces were blocked in the first two blocks, and the order of the two blocks was counterbalanced across participants. The last block was used for a manipulation check.

The first two blocks each included learning and test phases. In the learning phase, the participants were instructed to remember the faces in order to recognize them later. Ten faces were presented in random order. Moreover, each trial began with a 500-ms fixation cross; then a face with its semantic information was presented for $5,000 \mathrm{~ms}$, followed by a $500-\mathrm{ms}$ blank. After the learning phase, the participants were allowed a short break. In the test phase, the 20 faces without semantic information were sequentially presented in random order; among the 20 faces, 10 were old and 10 were new faces. Participants were asked to judge whether the presented face was an old or a new face and to press " $F$ " for old and " $J$ " for new. To counterbalance the old and the new faces, 10 new faces used for half the number of participants became old faces for the other half of participants.

To ensure that the semantic information developed in the pilot experiment was still effective for the participants in this experiment, in the third block, the participants had to rate to what extent the sentence below the face described the person's face. Each participant evaluated only the faces that had appeared in their own learning phase, but they rated both consistent and inconsistent descriptors for their appropriateness. The procedure was the same as that in the pilot study.

\section{Results and discussion}

The data of 8 participants were discarded because their accuracies in one of the race blocks were below .51 .
Fig. 1 Samples of stimuli in the learning phase for the control group, consistent group, and inconsistent group

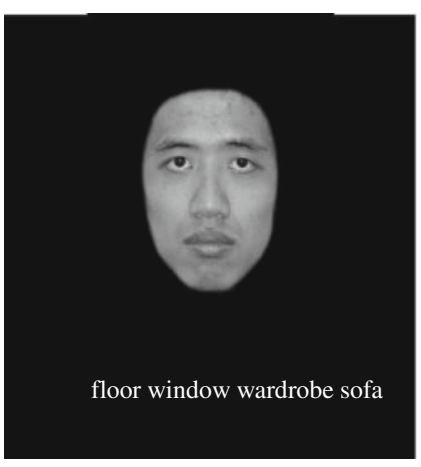

Control

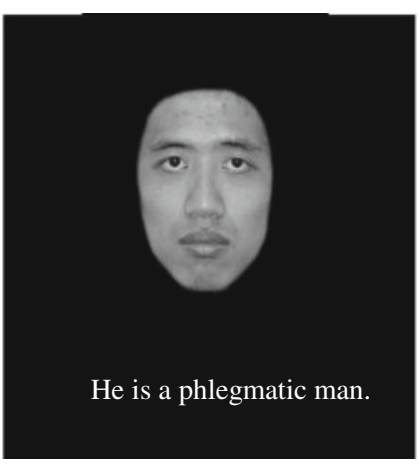

Consistent

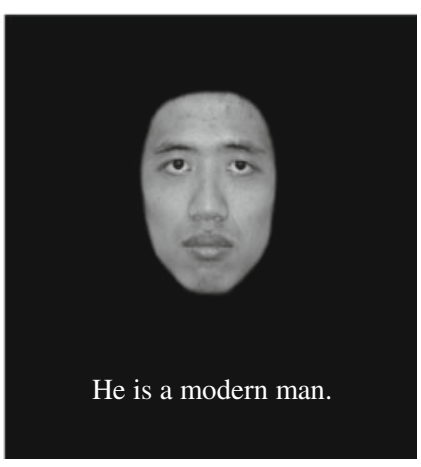

Inconsistent 
Manipulation check Responses in the manipulation check were analyzed as in the pilot study. One-sample $t$-tests, with the test value set as 3 , indicated that the consistency ratings were significantly larger than 3 for the own-race faces $(M=$ 3.44, $S D=0.50)$ and the other-race faces $(M=3.58, S D=$ $0.43), t \mathrm{~s}(83)>8, p \mathrm{~s}<.001$, and inconsistency ratings were significantly lower than 3 for the own-race faces $(M=2.42$, $S D=0.47)$ and the other-race faces $(M=2.55, S D=0.44)$, $t \mathrm{~s}(83)<-9, p \mathrm{~s}<.001$. These results indicated that the manipulation of the semantic information was effective.

Face memory The face recognition sensitivities $\left(d^{\prime}=Z\right.$ (hit $)-$ $Z(\mathrm{FA})$, correcting for conditions where accuracy was perfect by replacing the score with $1-1 / 2 N$ ) in each condition (see Fig. 2), were subjected to a $2 \times 2$ mixed-repeated ANOVA. The main effect of race was significant, $F(1,80)=5.84$,
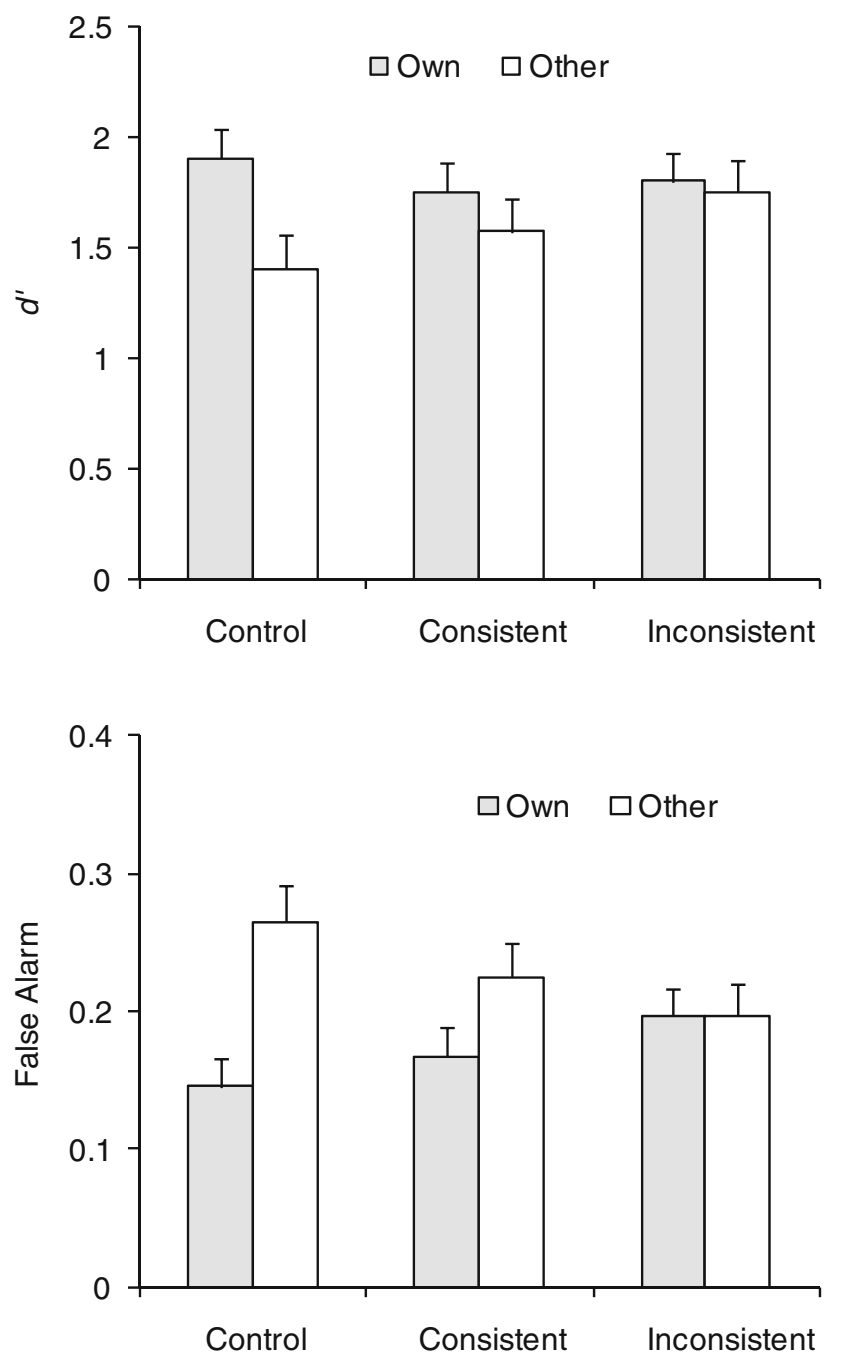

Fig. 2 Recognition sensitivity $d^{\prime}$ and false alarm for own-race (Asian) and other-race (Caucasian) faces under control (neutral semantic information), consistent (consistent semantic information), and inconsistent (inconsistent semantic information) conditions. Error bars represent the standard errors of the means $p=.018$, with better recognition for own-race $(M=1.82$, $S E=0.074)$ than for other-race $(M=1.58, S E=0.083)$ faces. The main effect of the semantic information was not significant, $F<1$. Neither was its interaction with race, $F(1,80)=1.739, p=.182$.

Because the ORE is often more pronounced for false alarms (FAs) than for hit rates, we also analyzed the hit rates and FA rates separately. The ANOVA results for hits showed no significant main effects or interactions, $F<1$. In contrast, the ANOVA results for FAs showed more FAs for other-race faces than for own-race faces, $F(1,80)=10.86$, $p<.001$. The interaction of race and group was also significant, $F(1,80)=3.697, p=.029$. A simple effect test showed that this deficiency in recognizing other-race faces existed in the control condition $(p<.001)$ and marginally in the consistent-information condition $(p=.066)$, but not in the inconsistent-information condition $(p=1.00)$. The main effect of semantic information was not significant, $F<1$.

\section{General discussion}

Consistent with previous research reports (Hugenberg et al., 2007; Lebrecht, Pierce, Tarr, \& Tanaka, 2009, Shriver \& Hugenberg, 2010), we found that individuation was helpful for improving the memory for faces and, thus, eliminating the ORE. However, this individuation effect was observed only in the inconsistent condition, but not in the consistent condition.

It is worth noting that unlike in our study, some previous researchers have failed to find reduction of the ORE following individuation of faces. For example, Devine and Malpass (1985) and Burgess and Weaver (2003) had participants judge whether a presented face was friendly. They found that this encoding improved memory performance for the faces; however, the improvement was similar for both own-race and other-race faces. Recently, Stahl, Wiese, and Schweinberger (2010), using attractiveness judgments to encourage individuation of the faces, and Rhodes, Locke, Ewing, and Evangelista (2009) had participants rate faces on how race typical they were; neither manipulation modulated the ORE. A common point in all these studies is that faces were judged on a single limited dimension (e.g., friendliness, attractiveness, typicality of race); therefore, not enough individual information for each face could be used for deep processing. Hence, the lack of the individuation effect in these studies may have been due to insufficient variability in the individuating information. Therefore, an effective individuation manipulation, such as an instruction to avoid the ORE (Hugenberg et al., 2007), individuated name-face training (Lebrecht et al., 2009), or the immediate accompaniment of inconsistent individual personality description for each face (as used in the present study), is necessary to promote other-race recognition performance and subsequently reduce the ORE. 
A potential explanation for the elimination of the ORE in the inconsistent condition was the amount of information that was presented, rather than individuation processes, because presentation of semantic information was different between the control conditions (a list of neutral words) and inconsistent (personality description) conditions. However, the lack of an effect for consistent information, which also used a personality description, ruled out this possibility. It appears to be the individuated association between the inconsistent personality description and faces, not the length or format of the description sentences, that affected face memory performance. Inconsistent description of faces may trigger deeper individual processing of faces than do consistent descriptions because the observer focuses attention on individuating features of the face in order to seek a resolution of the inconsistency. This process has stronger effects on recognition of other-race faces because people already have an innate tendency to individuate own-race faces (Hugenberg et al., 2007; Hugenberg \& Sacco, 2008; Hugenberg et al., 2010).
In summary, there are two contributions of the present study to the research literature of the ORE. First, we have provided further direct evidence of an individuation effect on the ORE. Second, we suggest this individuation effect size depends on the depth of the individuation processing. However, the present study has limitations for examining other alternative explanations (e.g., perceptual expertise hypothesis) of ORE. Further researches are needed to investigate whether and how perceptual expertise interacts with individuation processing and categorization processing.

Author Note We thank two reviewers for their constructive comments and Hongling Lao and Qiuzhen Lai for helping collect data.

This article was based on Lanya Zhang's thesis for her Master Degree. Lanya Zhang and Guomei Zhou made the same contribution to the article. This research was supported by the National Natural Science Foundation of China (30700230) to Guomei Zhou, and the Fundamental Research Funds for the Central Universities (09pywk22) to Guomei Zhou.

\section{Appendix}

Table 1 The 25 pairs of opposite words from the Big Five Personality Scale (Yang, McCrae, Costa, et al., 1999) and other 16 single words that are common descriptors of Chinese personality (Wang \& Cui, 2005)

\begin{tabular}{|c|c|c|}
\hline & 25 pairs words & 16 single words \\
\hline Stringent (迫切的) & calm (冷静的) & active (活跃的) \\
\hline Gregarious (群居的) & alone (独处的) & optimistic (乐观的) \\
\hline Dreamy (爱幻想的) & practical (现实的) & altruistic (利他的) \\
\hline Polite (礼貌的) & bold (粗鲁的) & scrupulous (严谨的) \\
\hline Tidy (整洁的) & untidy (混乱的) & determined (决断的) \\
\hline Cautious (谨慎的) & confident (自信的) & persistent (坚韧的) \\
\hline Optimistic (乐观的) & pessimistic (悲观的) & straightforward (爽直的) \\
\hline Theoretical (理论的) & practical (实践的) & large-minded (宽和的) \\
\hline Generous (大方的) & selfish (自私的) & nonchalant (淡泊的) \\
\hline Decisive (果断的) & open-minded (开放的) & social (合群的) \\
\hline Discouraged (泄气的) & optimistic (乐观的) & honest (诚信的) \\
\hline Explicit (外显的) & implicit (内隐的) & affectionate (重感情的) \\
\hline Follow imagination (跟从想像的) & obedient to authority (服从权威的) & sedate (沉稳的) \\
\hline Passionate (热情的) & phlegmatic (冷漠的) & ready-witted (机敏的) \\
\hline Of self-control (自制的) & apt to be interfered (易受干扰的) & patient (有耐性的) \\
\hline Apt to be embarrassed (易难堪的) & sophisticated (老练的) & enthusiastic (热情的) \\
\hline Sonsy (开朗的) & inhospitable (冷淡的) & \\
\hline Prefer novel ways (追求新奇的) & prefer routines (追求常规的) & \\
\hline Cooperative (合作的) & independent (独立的) & \\
\hline Prefer orders (喜欢次序的) & adapted to riproarious (适应喧闹的) & \\
\hline Apt to be distracted (易分心的) & self-possessed (镇静的) & \\
\hline Conservative (保守的) & thoughtful (有思想的) & \\
\hline Ambiguous (适于模棱两可的) & distinct (适于轮廓清楚的) & \\
\hline Trustful (信任的) & incredulous (怀疑的) & \\
\hline Punctual (守时的) & dilatory (拖延的) & \\
\hline
\end{tabular}




\section{References}

Ackerman, J. M., Shapiro, J. R., Neuberg, S. L., Kenrick, D. T., Becker, D. V., Griskevicius, V., et al. (2006). They all look the same to me (unless they're angry): From out-group homogeneity to out-group heterogeneity. Psychological Science, 17, 836-840.

Bernstein, M. J., Young, S. G., \& Hugenberg, K. (2007). The crosscategory effect: Mere social categorization is sufficient to elicit an own-group bias in face recognition. Psychological Science, $18,706-712$

Burgess, M. C. R., \& Weaver, G. E. (2003). Interest and attention in facial recognition. Perceptual and Motor Skills, 96, 467-480.

Crocker, J., Hanna, D. B., \& Weber, R. (1983). Person memory and causal attributions. Journal of Personality and Social Psychology, 44, 55-66.

Devine, P. G., \& Malpass, R. S. (1985). Orienting strategies in differential face recognition. Personality and Social Psychology Bulletin, 11, 33-40.

Hancock, K., \& Rhodes, G. (2008). Contact, configural coding and the other-race effect in face recognition. British Journal of Psychology, 99, 45-56.

Hastie, R., \& Kumar, P. A. (1979). Person memory: Personality traits as organizing principles in memory for behaviors. Journal of Personality and Social Psychology, 37, 25-38.

Hayward, W. G., Rhodes, G., \& Schwaninger, A. (2008). An own-race advantage for components as well as configurations in face recognition. Cognition, 106, 1017-1027.

Hills, P. J., Lewis, M. B., \& Honey, R. C. (2008). Stereotyping priming in face recognition: Interactions between semantic and visual information in face encoding. Cognition, 108, 185-200.

Hugenberg, K., Miller, J., \& Claypool, H. M. (2007). Categorization and individuation in the cross-race recognition deficit: Toward a solution to an insidious problem. Journal of Experiment Social Psychology, 43, 334-340.

Hugenberg, K., \& Sacco, D. F. (2008). Social categorization and stereotyping: How social categorization biases person perception and face memory. Social and Personality Psychology Compass, 2, 1052-1072.

Hugenberg, K., Young, S., Bernstein, M., \& Sacco, D. F. (2010). The categorization-individuation model: An integrative account of the cross race recognition deficit. Psychological Review, 117, 11681187.

Johnson, K. J., \& Fredrickson, B. L. (2005). "We all look the same to me": Positive emotions eliminate the own-race bias in face recognition. Psychological Science, 16, 875-881.

Klatzky, R. L., Martin, G. L., \& Kane, R. A. (1982). Semantic interpretation effects on memory for faces. Memory and Cognition, 10, 195-206.

Lebrecht, B., Pierce, L. J., Tarr, M. J., \& Tanaka, J. W. (2009). Perceptual other-race training reduces implicit racial bias. PloS One, 4, e4215.
Malpass, R. S., \& Kravitz, J. (1969). Recognition for faces of own and other race. Journal of Personality and Social Psychology, 13, 330-334.

Meissner, C. A., \& Brigham, J. C. (2001). Thirty years of investigating the own-race bias in memory for faces: A metaanalytic review. Psychology, Public Policy, and Law, 7, 3-35.

Michel, C., Caldara, R., \& Rossion, B. (2006). Same-race faces are perceived more holistically than other-race faces. Visual Cognition, 14, 55-73.

O'Sullivan, C. S., \& Durso, F. T. (1984). Effect of schema-incongruent information on memory for stereotypical attributes. Journal of Personality and Social Psychology, 47, 55-70.

Rahman, R. A., \& Sommer, W. (2008). Seeing what we know and understand: How knowledge shapes perception. Psychonomic Bulletin and Review, 15(6), 1055-1063.

Rhodes, G., Locke, V., Ewing, L., \& Evangelista, E. (2009). Race coding and the other-race effect in face recognition. Perception, $38,232-241$.

Rodin, M. J. (1987). Who is memorable to whom: A study of cognitive disregard. Social Cognition, 5, 144-165.

Sangrigoli, S., Pallier, C., Argenti, A.-M., Ventureyra, V. A. G., \& de Schonen, S. (2005). Reversibility of the other-race effect in face recognition during childhood. Psychological Science, 16, 440 444.

Shriver, E., \& Hugenberg, K. (2010). Power, individuation and the cross-race recognition deficit. Journal of Experimental Social Psychology, 46, 767-774.

Sporer, S. L. (2001). Recognizing faces of other ethnic groups: An integration of theories. Psychology, Public Policy, and Law, 7, 36-97.

Srull, T. K. (1981). Person memory: Some tests of associative storage and retrieval models. Journal of Experimental Psychology: Human Learning and Memory, 7, 440-463.

Srull, T. K., Lichtenstein, M., \& Rothbart, M. (1985). Associative storage and retrieval processes in person memory. Journal of Experimental Psychology. General, 11, 316-435.

Stahl, J., Wiese, H., \& Schweinberger, S. R. (2010). Learning task affects ERP-correlates of the own-race bias, but not recognition memory performance. Neuropsychologia, 48, 2027-2040.

Tanaka, J. W., Kiefer, M., \& Bukach, C. M. (2004). A holistic account of the own-race effect in face recognition: Evidence from a crosscultural study. Cognition, 93, B1-B9.

Wang, D. F., \& Cui, H. (2005). Exploring personality structure of the Chinese. Journal of Southwest China Normal University, 31, 516.

Wright, D. B., Boyd, C. E., \& Tredoux, C. (2003). Inter-racial contact and the own race bias for face recognition in South Africa and England. Applied Cognitive Psychology, 17, 365-373.

Yang, J., McCrae, R. R., Costa, P. T., Jr., Dai, X., Yao, S., Cai, T., et al. (1999). Cross-cultural personality assessment in psychiatric populations: The NEO-PI-R in the People's Republic of China. Psychological Assessment, 11, 359-368. 